\title{
Salvage high-dose-rate interstitial brachytherapy for perineal recurrence of prostate cancer after surgery and radiotherapy: a case report
}

\author{
Andrea Vavassori, MD!', Stefano Durante, MD!, Matteo Augugliaro, MDl, Stefania Comi, MSc², Raffaella Cambria, MSc², \\ Federica Cattani, MSc2, Roberta Lazzari, MD!, Deliu Victor Matei, MD³, Ottavio De Cobelli, MD³,4, Roberto Orecchia, MD5, \\ Barbara Alicja Jereczek-Fossa, MD, PhD1.4 \\ 'Department of Radiotherapy, IEO European Institute of Oncology IRCCS, Milan, Italy. 2Unit of Medical Physics, IEO European Institute of \\ Oncology IRCCS, Milan, Italy, ${ }^{3}$ Department of Urology, IEO European Institute of Oncology IRCCS, Milan, Italy, ${ }^{4}$ Department of Oncology \\ and Hemato-Oncology, University of Milan, Milan, Italy, ${ }^{5}$ Scientific Directorate, IEO European Institute of Oncology IRCCS, Milan, Italy
}

\begin{abstract}
Perineal seeding of tumor cells from prostate cancer (PCa) is very rare, and no standard treatment exists for this atypical presentation with no evidence of distant metastases. Local excision or external beam radiotherapy are used as local salvage treatments for such perineal masses, including those occurring after biopsy, surgery, or interstitial brachytherapy.

We report on a patient who presented no evidence of disease and no late urinary or gastrointestinal toxicities at 58 months after receiving high-dose-rate brachytherapy (HDR-BT) for perineal recurrence of PCa after radical prostatectomy and salvage external beam radiotherapy. To the best of our knowledge, this is the first case treated with HDR-BT in this scenario.

J Contemp Brachytherapy 2020; 12, 5: 492-496 DOI: https://doi.org/10.5114/jcb.2020.100383
\end{abstract}

Key words: interstitial HDR brachytherapy, perineal prostate cancer recurrence, reirradiation.

\section{Purpose}

Perineal seeding of tumor cells from prostate cancer (PCa) is very rare and no standard treatment exists for this atypical presentation with no evidence of distant metastases, including those occurring after biopsy, surgery, cryotherapy, or interstitial brachytherapy (BT) $[1,2,3,4,5,6,7]$. Local excision is commonly used as local salvage treatments for such perineal masses. Stereotactic body radiotherapy (SBRT) was investigated in selected cases, especially for non-operable patients (medical contraindications or patient's refusal) and for those with lesions at high-risk of poor functional results after surgery.

\section{Case report}

In 2015, a 78-year-old man was admitted to our Radiotherapy Department for a perineal clinical recurrence of PCa after surgery and salvage external beam radiotherapy (EBRT). In April 2010, based on a positive digital rectal exam (DRE) in the left prostatic lobe, which was performed for a low urinary tract obstructive syndrome, the patient underwent (in another hospital) a transrectal ultrasound (TRUS)-guided biopsy, in spite of $0.54 \mathrm{ng} / \mathrm{ml}$ prostate specific antigen (PSA) level. The pathology revealed a prostatic adenocarcinoma, Gleason score (GS) $3+5$ with perineural invasion. As the serum PSA was low, neuroendocrine pattern was suspected, but serum chromogranin A (CgA) was normal $(68.3 \mathrm{ng} / \mathrm{ml})$. No data about serum neuron-specific enolase (NSE) was available. Both staging $\mathrm{CT}$ and bone scans resulted negative. As a consequence, a radical prostatectomy was proposed and upon patient's acceptance, the procedure was carried out in June 2010. Final pathology showed a bilateral adenocarcinoma GS $5+4$ with perineural invasion, pT2c pN0 (0/14 pelvic nodes), negative surgical margins, but extended at less than 1-mm from the surgical margin at the apex. At 30 days after surgery, the PSA was 0, while serum CgA verified a time limited increase to $150.9 \mathrm{U} / 1$ and then dropped to $80.5 \mathrm{U} / 1$. Two years later, repeated pelvic computed tomography (CT) scan and bone scintigraphy showed no evidence of distant metastasis, but choline positron emission tomography (PET)/CT scan revealed a pathologic uptake in a nodule of $18-\mathrm{mm}$, placed posteriorly to the vesical-urethral anastomosis, with serum PSA $0.02 \mathrm{ng} / \mathrm{ml}$.

Address for correspondence: Stefano Durante, MD, Department of Radiotherapy, IEO European Institute Received: 25.05.2020 of Oncology IRCCS, via Ripamonti 435, 20141 Milan, Italy, phone: +39 0257483037, 
TRUS-guided biopsy was planned, and pathology revealed a poorly differentiated adenocarcinoma, GS $5+4$, with fibromuscular and perineural infiltration. The patient was referred to our uro-oncology service, and in May 2012, total androgen deprivation therapy (ADT) with bicalutamide and triptorelin was started. Successively, in another hospital, salvage EBRT was scheduled and performed between May and July 2012, delivering a total dose of 70 Gy in 35 fractions (2 Gy daily fractions, 5 fractions/week). The treatment was well tolerated without any significant acute and late urinary or intestinal toxicity. Follow-up was negative until November 2014, when a choline PET/CT scan and a pelvic multiparametric magnetic resonance imaging (MRI) showed a new recently onset of pseudo-nodular lesion in the perineum (Figure 1). Fusion ultrasound (US)/MRI-targeted biopsy was carried out showing a grade 3 prostatic adenocarcinoma with necrosis. No data about serum NSE, nor CgA were available. The multidisciplinary tumor board proposed to evaluate the feasibility of a reirradiation with BT. The diagnostic work-up, with repeated pelvic multiparametric MRI and choline PET/CT scan evidenced that this was the case of a local failure: a perineal nodular lesion $(22 \times$ $16 \times 9 \mathrm{~mm}$ ) placed caudally to the urethro-vesical anastomosis, strictly adherent to the bulbar urethra, and to the external anal sphincter, but without distant metastasis. After a preliminary clinical evaluation and a preplanning TRUS, high-dose-rate brachytherapy (HDR-BT) treatment was confirmed and planned in June 2015. Before processing the treatment, a written informed consent for the treatment and for using his anonymized clinical data and images for research and educational purposes was obtained from the patient. This study is a part of research notified to our institutional ethical committee (notification No. 729).

Under epidural anesthesia and after a Foley catheter positioning, 14 plastic afterloading needles (NucletronElekta) were inserted in the nodular lesion and in the surrounding tissue using a transperineal and TRUS-guided approach. The needles were held in place using a dedicated perineal template (prostate stepper template set, Nucletron-Elekta, The Netherlands).

Once the implantation was completed, a CT scan with $2.5 \mathrm{~mm}$ slice thickness and separation was performed and entered into the BT treatment planning system (Oncentra Brachy Planning, Nucletron-Elekta, The Netherlands), allowing reconstruction of the catheters, definition of the clinical target volume (CTV $=$ perineal nodule), the planning target volume (PTV $=$ CTV plus $5 \mathrm{~mm}$ margin), and organs at risk (rectum, anal canal, bladder and urethra).

Computerized optimization of dwell positions and times of stepping source was performed for fine tuning of the isodose distributions (Table 1 )

Table 1. DVH parameters for clinical target volume (CTV), planning target volume (PTV), and organs at risk (OARs)

\begin{tabular}{lc} 
Parameter & \\
\hline CTV & $114 \%(5.12 \mathrm{~Gy})$ \\
\hline CTV $_{90}$ & $99.5 \%$ \\
\hline CTV $V_{100}$ & $16 c c$ \\
\hline PTV & $91.2 \%(4.1 \mathrm{~Gy})$ \\
\hline PTV $\mathrm{D}_{90}$ & $83.2 \%$ \\
\hline PTV $\mathrm{V}_{100}$ & $7.62 \%(0.3 \mathrm{~Gy})$ \\
\hline $\mathrm{D}_{2 c c}$ bladder & $20.4 \%(0.9 \mathrm{~Gy})$ \\
\hline$D_{2 c c}$ urethra & $11.8 \%(0.53 \mathrm{~Gy})$ \\
\hline$D_{2 c c}$ rectum & $31.4 \%(1.4 \mathrm{~Gy})$
\end{tabular}

$D_{90}$ - dose to the $90 \%$ of volume, $V_{100}$ - volume encompassed by the $100 \%$ isodose, $D_{2 c c}$-dose to $2 c c$ of the organ


Fig. 1. Pre-brachytherapy MRI (A) and choline PET (B) 

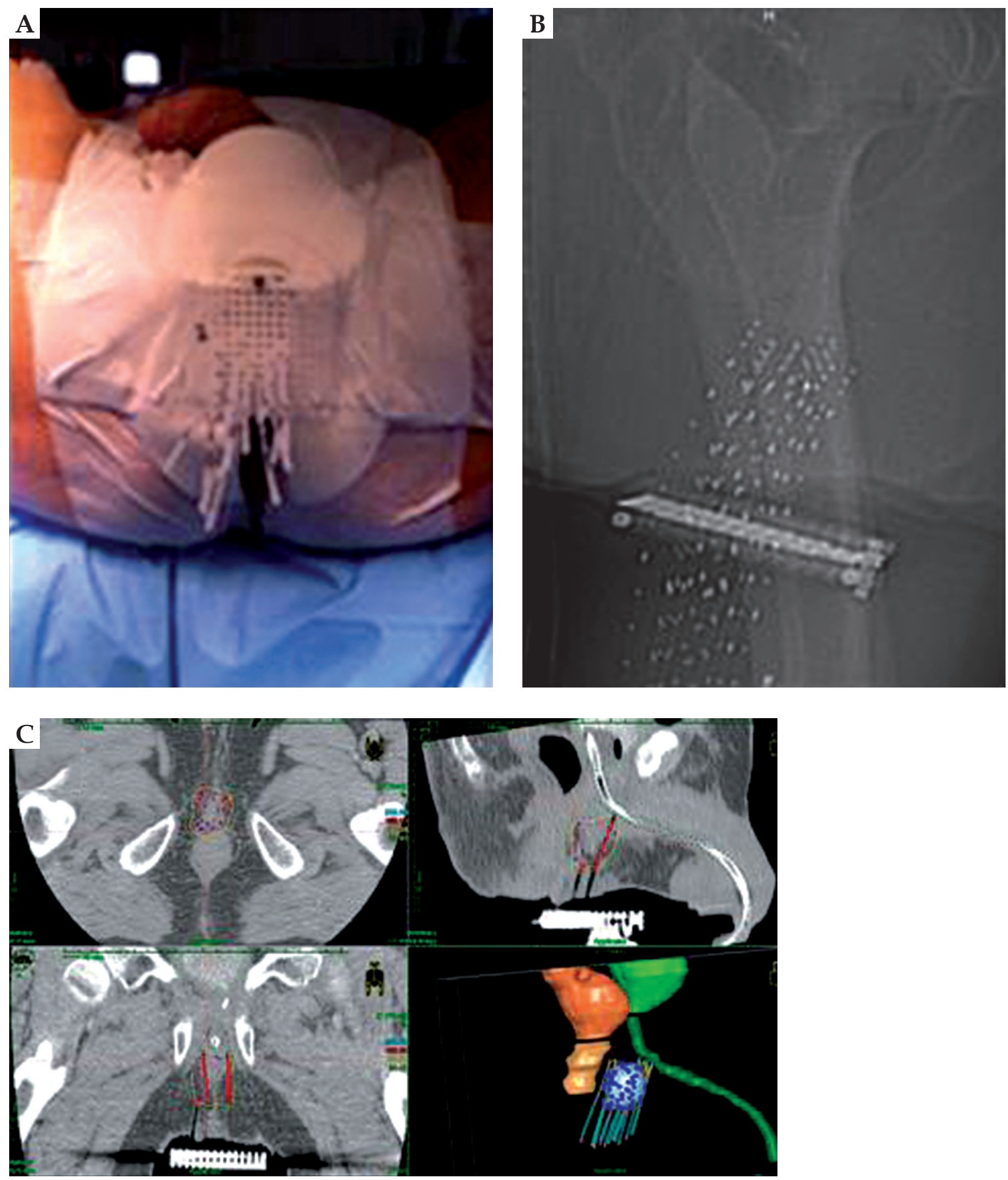

Fig. 2. Implant (A, B) and treatment plan (C)

Fractionated HDR-BT was delivered using an HDR afterloader (MicroSelectron, Nucletron-Elekta, The Netherlands) containing a single iridium-192 ( $\left.{ }^{192} \mathrm{Ir}\right)$ source. Before the administration of each treatment fraction, the implant was examined by a radiation therapist and radiation oncologist to ensure no critical displacement of the needles (Figure 2).
A total dose of 27 Gy in 6 fractions, 2 fractions per day, with a minimum interval of $6 \mathrm{~h}$ was administrated in 3 consecutive days. At the end of last fraction, the template and implant needles were removed. Three months after HDR-BT, the androgen deprivation therapy was terminated. During follow-up time, a routine PSA testing, pelvic MRI, choline PET/CT scan, and clinical examina- 


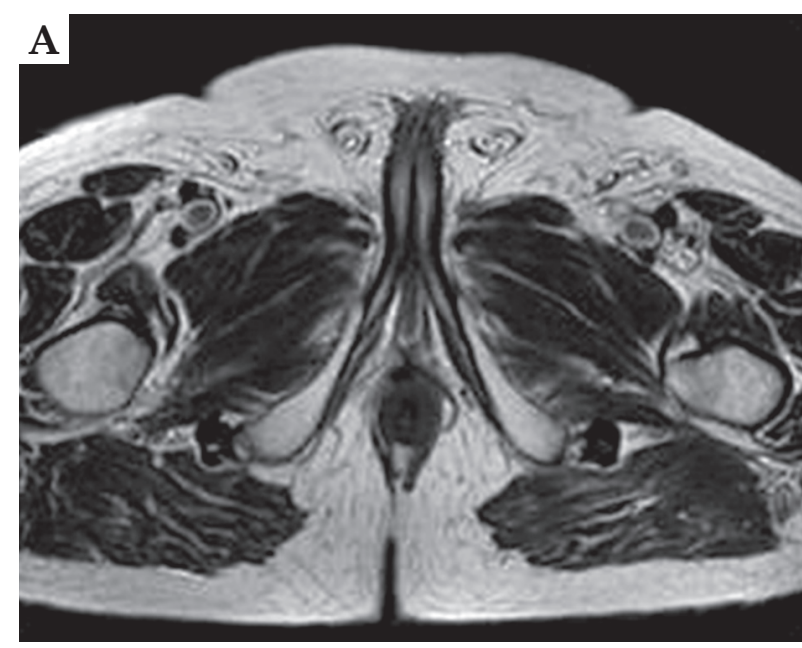

Fig. 3. Follow-up at 2 years with axial (A) and sagittal (B) MRI

tion were performed to assess local control (Figure 3). Acute and late side effects were classified according to the Radiation Therapy Oncology Group (RTOG) and the European Organization for Research and Treatment of Cancer (EORTC) scale [8]. No severe acute and late rectal or urinary toxicities was reported (no urgency, no incontinence, no dysuria). Three years after HDR-BT, CgA was $28.5 \mathrm{ng} / \mathrm{ml}$. The patient has no evidence of biochemical failure (PSA, $0 \mathrm{ng} / \mathrm{ml}$; testosterone, $1.1 \mathrm{ng} / \mathrm{ml}$ ) or clinical recurrence at 58 months after the salvage treatment.

\section{Discussion}

Due to recent advances in imaging and radiotherapy techniques, it is possible to detect small lesions and irradiate them with high doses in few fractions or removing them surgically [9]. Many factors may influence the therapeutic choice, and a multidisciplinary approach is mandatory since no guidelines are available. Salvage treatment in locally relapsed PCa depends on the recurrence site, patient's age, performance status, medical comorbidities, potential impact on quality of life, and patient's expectations and preferences as well as the available technology $[10,11,12]$. Perineal recurrence of PCa is a rare complication after biopsy, surgery, brachytherapy, or cryotherapy due to seeding of malignant cells. Only few cases are described in the literature, and at the moment, there are no clinical recommendations for prevention or management of this rare clinical condition $[1,2,3,4,5,6]$. In 1989, Moul et al. analyzed more than 2,000 perineal prostate biopsies. Among the group of 502 patients with PCa, they described 5 cases of perineal seeding $(1 \%)$, with distant metastases (concomitant or within 16 months) in each case [2]. In a recent review, Volanis et al. identified 26 articles reporting 42 patients (incidence $<1 \%$ ) with needle tract seeding after transperineal or transrectal prostate biopsy. They found no correlation between the develop-



ment of seeding and the type or diameter of needle used for biopsy in any of the reviewed papers [5]. Recently, due to widespread of robotic surgery for organ-confined PCa treatment, port site recurrence in patients undergoing RALP have been published. Cell spillage and immunosuppression may favor this pattern of recurrence [13].

As far as the therapeutic approach is concerned, only one reported patient with a perineal recurrence, following a permanent BT received stereotactic radiotherapy with volumetric modulated arc therapy (VMAT) to a dose of 35 Gy in 5 fractions. Two gold markers were implanted into the nodule to ensure image guidance. One year after treatment, the patient remained asymptomatic and free of disease [9]. Early detection of these disease recurrences is mandatory to intercept the oligometastatic status, which is still suitable for local therapeutic approach. To the best of our knowledge, this is the first case of patient treated with HDR-BT, and future studies are required to determine the best treatment in these patients.

\section{Conclusions}

Evidence from studies comparing the results of radiation therapy with other treatments in perineal PCa recurrence is lacking in the literature due to the infrequent presentation of this disease relapse. Despite the several limitations of a single case report, our data support the concept that HDR-BT for the treatment of perineal nodular lesions is feasible and effective.

\section{Acknowledgements}

This work was partially supported by the Italian Ministry of Health, with Ricerca Corrente and $5 \times 1,000$ funds.

\section{Disclosure}

The authors report no conflict of interest. 


\section{References}

1. Haddad FS, Somsin AA. Seeding and perineal implantation of prostatic cancer in the track of the biopsy needle: three case reports and a review of the literature. J Surg Oncol 1987; 35: 184-191.

2. Moul JW, Miles BJ, Skoog SJ et al. Risk factors for perineal seeding of prostate cancer after needle biopsy. J Urol 1989; 142: 86-88

3. Moul JW, Bauer JJ, Srivastava S et al. Perineal seeding of prostate cancer as the only evidence of clinical recurrence 14 years after needle biopsy and radical prostatectomy: molecular correlation. Urology 1998; 51: 158-160.

4. Downey DB, Chin JL, Williams JC. Perineal prostate cancer seeding along needle tract following cryosurgery. Can J Urol 1999; 6: 823-825.

5. Volanis D, Neal DE, Warren AY et al. Incidence of needletract seeding following prostate biopsy for suspected cancer: a review of the literature. BJU Int 2015; 115: 698-704.

6. Teh BS, Chou CC, Schwartz MR, et al. Perineal prostatic cancer seeding following Radioactive seed brachytherapy. J Urol 2001; 166: 212.

7. Cooper S, Pillinger T, Ahmed I, et al. Perineal recurrence of prostate cancer post-brachytherapy. BJR Case Rep 2019; 5: 20180104.

8. Cox JD, Stetz J, Pajak TF. Toxicity criteria of the Radiation Therapy Oncology Group (RTOG) and the European Organization for Research and Treatment of Cancer (EORTC). Int J Radiat Oncol Biol Phys 1995; 31: 1341-1346.

9. Eppinga W, Vijverberg $P$, Moerland $R$ et al. Perineal recurrence of prostate cancer six years after trans-perineal brachytherapy. J Contemp Brachytherapy 2015; 6: 386-388.

10. De Cicco L, Vavassori A, Cattani F et al. Salvage high dose rate brachytherapy after primary external beam irradiation in localized prostate cancer: a case report. Tumori 2009; 95: 553-556.

11. Jereczek-Fossa BA, Rojas DP, Zerini D et al. Reirradiation for isolated local recurrence of prostate cancer: Mono-institutional series of 64 patients treated with salvage stereotactic body radiotherapy (SBRT). Br J Radiol 2019; 92: 20180494.

12. Volpe S, Jereczek-Fossa BA, Zerini D et al. Case series on multiple prostate re-irradiation for locally recurrent prostate cancer: something ventured, something gained. Neoplasma 2019; 66: 308-314.

13. Calderoni L, Matei Deliu V, Farolfi A et al. Single subcutaneous prostate cancer metastasis detected by ${ }^{68} \mathrm{Ga}$-PSMA PET/CT during early biochemical relapse: a case report. Clin Genitourin Cancer 2019; 17: e356-e359. 